\title{
Overdoping Graphene beyond the van Hove Singularity
}

\author{
Philipp Rosenzweig $\odot,{ }^{1, *}$ Hrag Karakachian $\odot,{ }^{1}$ Dmitry Marchenko $\odot,{ }^{2}$ Kathrin Küster $\odot,{ }^{1}$ and Ulrich Starke ${ }^{1}$ \\ ${ }^{1}$ Max-Planck-Institut für Festkörperforschung, Heisenbergstraße 1, 70569 Stuttgart, Germany \\ ${ }^{2}$ Helmholtz-Zentrum Berlin für Materialien und Energie, \\ Elektronenspeicherring BESSY II, Albert-Einstein-Straße 15, 12489 Berlin, Germany
}

(Received 14 July 2020; accepted 9 September 2020; published 19 October 2020)

\begin{abstract}
At very high doping levels the van Hove singularity in the $\pi^{*}$ band of graphene becomes occupied and exotic ground states possibly emerge, driven by many-body interactions. Employing a combination of ytterbium intercalation and potassium adsorption, we $n$ dope epitaxial graphene on silicon carbide past the $\pi^{*}$ van Hove singularity, up to a charge carrier density of $5.5 \times 10^{14} \mathrm{~cm}^{-2}$. This regime marks the unambiguous completion of a Lifshitz transition in which the Fermi surface topology has evolved from two electron pockets into a giant hole pocket. Angle-resolved photoelectron spectroscopy confirms these changes to be driven by electronic structure renormalizations rather than a rigid band shift. Our results open up the previously unreachable beyond-van-Hove regime in the phase diagram of epitaxial graphene, thereby accessing an unexplored landscape of potential exotic phases in this prototype two-dimensional material.
\end{abstract}

DOI: 10.1103/PhysRevLett.125.176403

Graphene, a honeycomb monolayer of carbon atoms, has been extensively studied in view of its two-dimensional (2D) massless Dirac fermions [1-5]. Recently, the focus has shifted towards inducing correlated phases in this prototype 2D material $[6,7]$. The strategy is to increase the density of states at the Fermi level $E_{F}$, thus boosting many-body interactions and instabilities towards the pursued ground states. Possible routes are band structure engineering in twisted graphene bilayers [8-11] or flat band formation in epitaxial bilayer graphene [12]. Alternatively, excessive $n$ doping of quasi-free-standing monolayer graphene (QFMLG) on $\mathrm{SiC}$ pushes the $\pi^{*}$ van Hove singularity (VHS) into the vicinity of $E_{F}$ [13-15]. There, many-body interactions warp the pointlike VHS into a flat band pinned to $E_{F}$ along the $\overline{\mathrm{KMK}^{\prime}}$ Brillouin zone (BZ) border: an extended van Hove scenario, reminiscent of high- $T_{c}$ superconductors [16-21]. Upon reaching the VHS, the Fermi surface (FS) undergoes a Lifshitz transition [22,23] whereby its topology changes from two electron pockets into a single hole pocket [13-15]. Concomitantly, theory predicts various ordered ground states such as chiral superconductivity or charge density wave [24-30]. The exact filling factors of the $\pi^{*}$ band at which the individual phases may stabilize are however hard to pinpoint in calculations, further

Published by the American Physical Society under the terms of the Creative Commons Attribution 4.0 International license. Further distribution of this work must maintain attribution to the author(s) and the published article's title, journal citation, and DOI. Open access publication funded by the Max Planck Society. complicated by the severe band structure renormalizations which have not been taken into account until recently [15]. Experimentally, a tunable $n$-type doping near van Hove filling is therefore desirable. While the carrier density can be reduced away from the VHS [14], overdoping has not yet been unambiguously confirmed and remains an important task at the basis of exploring the phase diagram of highly doped graphene. As more and more electrons are transferred onto QFMLG, the $\pi^{*}$ hole pocket in the FS will naturally shrink [Fig. 1(a), bottom], providing direct evidence for overdoping past the Lifshitz transition.

Here, we $n$ dope epitaxial graphene on $\mathrm{SiC}$ to beyond the $\pi^{*}$ VHS. Carrier densities of up to $5.5 \times 10^{14} \mathrm{~cm}^{-2}$ are achieved via ytterbium intercalation of the $(6 \sqrt{3} \times$ $6 \sqrt{3}) \mathrm{R} 30^{\circ}$ carbon buffer layer and subsequent potassium deposition. Angle-resolved photoelectron spectroscopy (ARPES) reveals a shrinking $\pi^{*}$ hole pocket, unambiguously confirming the completion of the Lifshitz transition. The latter is driven by renormalizing $\pi$ bands rather than a rigid band shift, demonstrating the extensive influence of many-body interactions at extreme doping levels. Upon overdoping a previously inaccessible regime is reached in the phase diagram of graphene, where exotic ordered ground states might emerge [24-30].

The $(6 \sqrt{3} \times 6 \sqrt{3}) \mathrm{R} 30^{\circ}$ carbon buffer layer was grown on $6 \mathrm{H}-\mathrm{SiC}(0001)$ following Emtsev et al. [31]. Its transformation into QFMLG via intercalation of ytterbium at a sample temperature of $250^{\circ} \mathrm{C}$ is detailed elsewhere [14]. The QFMLG samples were transferred to the synchrotron facility in an ultrahigh vacuum suitcase (Ferrovac $\mathrm{GmbH}$ ) at a pressure $<1 \times 10^{-9}$ mbar, ensuring sample stability such that no additional annealing was necessary. ARPES 

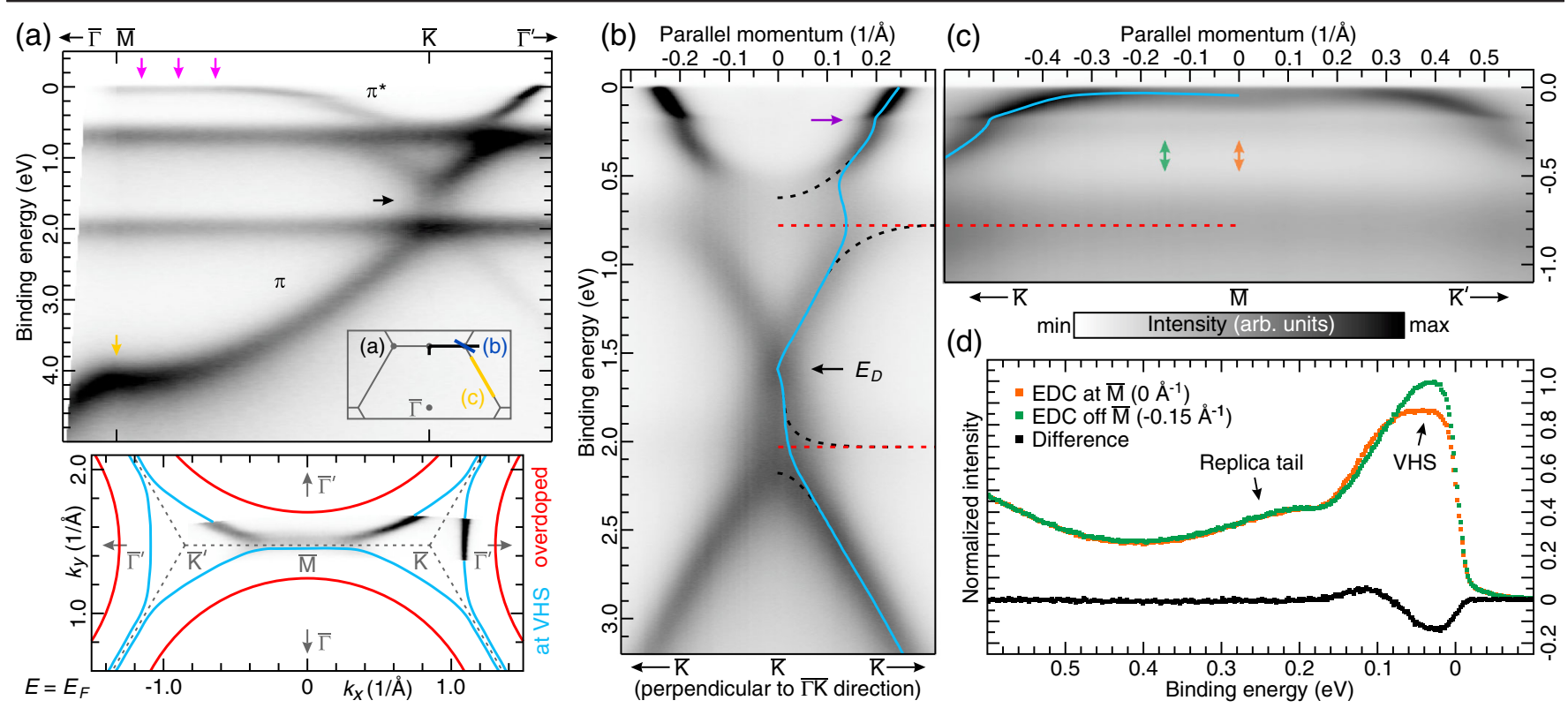

FIG. 1. (a) Dispersion along $\overline{\Gamma M K}$ (top) and FS (bottom) of Yb-intercalated graphene ( $h \nu=80 \mathrm{eV})$. The inset shows the orientations of the datasets (a)-(c). Solid curves in the bottom panel indicate the FS hole pocket at van Hove filling (blue curves) and beyond (red curves, exaggerated for clarity). (b),(c) High-resolution energy-momentum cuts $(h \nu=40 \mathrm{eV}$ ) recorded (b) at $\overline{\mathrm{K}}$ perpendicular to $\overline{\Gamma \mathrm{K}}$ and (c) along $\overline{\mathrm{KMK}}^{\prime}$. Half of the respective data are overlayed with solid blue curves tracking the spectral maxima of the graphene $\pi$ bands which hybridize (dashed black guides to the eye) with the Yb $4 f$ states (dashed red lines) near $\overline{\mathrm{K}}$. (d) EDCs extracted from (c) at $\overline{\mathrm{M}}$ (0 $\AA^{-1}$, orange dots) and $0.15 \AA^{-1}$ away (green dots) together with their difference curve (black dots).

was performed at the $1^{2}$ endstation of the UE112 beamline at BESSY II, Helmholtz-Zentrum Berlin [32] using linearly polarized synchrotron radiation and a hemispherical analyzer (Scienta R8000) with a 2D electron detector. The angular resolution was $0.1^{\circ}$ while the maximally achieved energy resolution of the entire setup (beamline and analyzer) was $20 \mathrm{meV}$. During all experiments the sample was kept at $\approx 20 \mathrm{~K}$ and the base pressure was $<5 \times 10^{-10}$ mbar. Potassium was deposited in situ on the cold sample from a commercial alkali metal dispenser (SAES Getters; operated at 5.6 A, $1.3 \mathrm{~V}$ ).

We first set a basis for our overdoping study by presenting high-resolution, low-temperature ARPES of the extended van Hove scenario in pristine $\mathrm{Yb}$-intercalated graphene. The top panel of Fig. 1(a) gives an overview of the dispersion along $\overline{\Gamma \mathrm{MK}}$, probed at a photon energy of $h \nu=80 \mathrm{eV}$. Nondispersive $\mathrm{Yb} 4 f$ states at $\approx 0.8$ and $\approx 2.0 \mathrm{eV}$ below $E_{F}$ are superimposed on the graphene $\pi$ bands. Their conical dispersions near $\overline{\mathrm{K}}$ and $\overline{\mathrm{K}}^{\prime}$ (black arrow) are connected by the lower $\pi$ band through a saddle point $\approx 4.1 \mathrm{eV}$ below $E_{F}$ at $\overline{\mathrm{M}}$ (yellow arrow). Because of strong $n$ doping the $\pi^{*}$ band becomes occupied to a large extent and its $\bar{M}$-point VHS reaches $E_{F}$. Contrary to the lower saddle point, the $\pi^{*}$ VHS is warped into a flat band pinned to $E_{F}$ over a wide $\left(\approx 0.6 \AA^{-1}\right)$ momentum range along $\overline{\mathrm{KMK}}^{\prime}$ (magenta arrows), generating a FS hole pocket around $\bar{\Gamma}$ [Fig. 1(a) bottom]-the extended van Hove scenario [13-21]. In a single-particle picture [5] the Dirac point $\left(E_{D}\right)$ should lie halfway between the lower and upper saddle points, i.e., about $2.0 \mathrm{eV}$ below $E_{F}$. However, $\left|E_{F}-E_{D}\right| \approx 1.6 \mathrm{eV}$ in our data. Instead of a purely rigid shift, the $\pi^{*}$ band bends down and reduces its bandwidth at $\overline{\mathrm{M}}$ by more than $1 \mathrm{eV}$ (cf. Refs. [5,33]), therefore reaching the VHS at an electron density on the order of $3 \times 10^{14} \mathrm{~cm}^{-2}$ [14] (cf. below).

Figure 1(b) shows a cut through the Dirac cone at $\overline{\mathrm{K}}$ perpendicular to $\overline{\Gamma K}(h \nu=40 \mathrm{eV})$. The solid blue curve tracks track the spectral maxima of the momentum distribution curves (MDCs). We identify the Dirac point (the branch crossing) at a binding energy of $E_{D}=1.58 \pm 0.02 \mathrm{eV}$, confirming with higher accuracy its previous determination [14]. The conical dispersion of graphene is distorted around the $\mathrm{Yb} 4 f$ states (dashed red lines). This becomes most obvious near the $4 f_{7 / 2}$ level at a binding energy of $0.78 \pm 0.01 \mathrm{eV}$ where the group velocity of the $\pi^{*}$ band seems to change sign. This unphysical MDCderived dispersion strongly suggests an anticrossing-type $\pi-4 f$ hybridization (dashed black guides to the eye) - a scenario supported by band structure calculations for $\mathrm{Yb}$ sandwiched in between a freestanding graphene bilayer [34]. The prominent kink $0.19 \pm 0.01 \mathrm{eV}$ below $E_{F}$ (purple arrow) can be ascribed to electron-phonon interaction $[33,35,36]$. From the slope of the real part of the spectral function near $E_{F}$ [37] we estimate a total coupling strength $\lambda \approx 0.4$, compatible with previous studies of highly $n$-doped graphene on $\mathrm{SiC}[34,38]$.

Figure 1(c) tracks the $\pi^{*}$ dispersion along $\overline{\mathrm{KMK}}^{\prime}$. The solid blue curve is fitted to the MDC (for binding 
energies $>0.1 \mathrm{eV}$ ) and energy distribution curve (EDC, for parallel momenta $\left|k_{\|}\right|<0.45 \AA^{-1}$ ) maxima. Interestingly, the flat band seems to disperse very slightly $(<20 \mathrm{meV})$ through a local minimum at $\overline{\mathrm{M}}$. This tiny dip might be a consequence of the changing EDC line shape from the edge towards the center of the flat band [Fig. 1(d)]. The VHS signature in the EDC at $\overline{\mathrm{M}}\left(0 \AA^{-1}\right.$, orange line $)$ is slightly broader, less intense and peaks into an $\approx 40 \mathrm{meV}$ wide plateau as compared to the sharper EDC at $-0.15 \AA^{-1}$ (green line) (cf. black difference curve). Linewidth broadening of the flat band along $\overline{\mathrm{KMK}}^{\prime}$, suggesting a varying quasiparticle scattering rate, has not yet been reported in similar systems $[13,15]$. Naively, one would expect the scattering probability to peak right at $\overline{\mathrm{M}}$ if the $\pi^{*}$ VHS were still pointlike. In turn, our data suggest that the $\bar{M}$ point's singular character is partially retained despite the extension of the VHS in $k$ space. The decreasing intensity of the flat band towards $\bar{M}$ could be explained by spectral weight redistribution upon VHS extension and linewidth broadening. While being also reminiscent of pseudogap formation as encountered in other correlated materials $[39,40]$, only temperature-dependent studies at ultimate resolution might shed light on this issue. The flat band at $E_{F}$ is accompanied by a replica $\approx 0.2 \mathrm{eV}$ below, which seems to emerge out of the kinks at $\pm 0.5 \AA^{-1}$ in Fig. 1(c) and whose width and intensity do not vary significantly along $\overline{\mathrm{KMK}}^{\prime}$ [Fig. 1(d)]. The same tail exists in the VHS regime of alkali-metal-doped [13] as well as Gdintercalated graphene [15] and was ascribed to polaron formation resulting from the coupling to optical phonons of graphene at $\bar{\Gamma}$. A coupling constant $\lambda_{\bar{\Gamma}} \approx 1$ to this specific mode was estimated in Ref. [15], which remains a reasonable value in the present case considering the similar intensity ratio $(\approx 2.2)$ between the VHS peak and the plateau $0.2 \mathrm{eV}$ below. These resemblances suggest that the feature is intrinsic to the extended van Hove scenario of graphene and, apart from the necessary charge transfer, independent of the respective dopants. Apparently, $\lambda_{\bar{\Gamma}}$ is strongly enhanced compared to graphene outside the VHS regime [41]. This is compatible with the hole pockets of adjacent BZs running parallel and very close to each other over a wide range along $\overline{\mathrm{KMK}^{\prime}}$ [Fig. 1(a) bottom] such that FS nesting will promote resonant coupling to the aforementioned zone-center phonons [15]. While the flat band formation (not its replica) was linked to spin fluctuations within graphene [15], charged impurity scatteringpromoted via a disordered intercalant [14]—could also account for $\pi^{*}$ band flattening, pronounced kinks along $\overline{\mathrm{KMK}}^{\prime}$, and a flat band replica near the kink energy [42]. The observed features might therefore emerge from a combination of these different mechanisms.

Figure 2(a) displays the FS of Yb-intercalated graphene, covering large portions of the first and adjacent BZs $(h \nu=80 \mathrm{eV})$. Entailed by the extended van Hove scenario, the two electron pockets centered at $\overline{\mathrm{K}}$ and $\overline{\mathrm{K}}^{\prime}$ demonstrate strong triangular warping and merge into a giant hole pocket around $\bar{\Gamma}$ via a Lifshitz transition $[22,23]$. The FS is quantified by fitting the MDCs in $k_{x}$ direction for $\left|k_{y}\right|<0.85 \AA^{-1}$. From the contour around $\bar{\Gamma}^{\prime}$ in the repeated BZ [blue curves in Figs. 2(a) and 2(e)] and following Luttinger's theorem $[43,44]$ we infer an electron density of $n=(3.66 \pm 0.40) \times 10^{14} \mathrm{~cm}^{-2}$ [45]. Being separated by only $\approx 0.02 \AA^{-1}$ from the $\overline{\mathrm{KMK}}^{\prime}$ border, the individual holelike contours of adjacent BZs cannot be unambiguously discerned, i.e., the VHS remains pinned to $E_{F}$ and the system is held right at the Lifshitz transition. Upon additional charge transfer onto QFMLG (i.e., overdoping), the completion of the Lifshitz transition could be directly visualized by a shrinking hole pocket [Fig. 1(a) bottom]. We thus performed sequential, 5-min-long deposition cycles of potassium, a well-known $n$-type dopant for epitaxial graphene [46]. After the first dosage the distance between the hole contour and the $\overline{\mathrm{KMK}^{\prime}}$ line has increased by a factor of 2.5 to $\approx 0.05 \AA^{-1}$ [purple curves in Figs. 2(b) and 2(e)] and the FS fit confirms an elevated carrier density of $n=(4.63 \pm 0.20) \times 10^{14} \mathrm{~cm}^{-2}$. Note also the suppression of the $\pi^{*}$ band inside the first BZ $\left(k_{x}<0\right)$ due to matrix-element effects (dark corridor) [47] and the deteriorating signal-to-noise ratio following potassium adsorption. After $10 \mathrm{~min}$ of exposure the hole pockets have shrunk to such extent that their individual contours are clearly distinguishable across the $\overline{\mathrm{KMK}}^{\prime}$ borders and the Lifshitz transition has unambiguously concluded at a charge carrier density of $n=(5.46 \pm 0.20) \times 10^{14} \mathrm{~cm}^{-2}$ [Fig. 2(c)]. Finally, during a third deposition cycle, the FS does no longer change significantly, indicating a saturation of doping within the error range [Fig. 2(d), $n=(5.54 \pm 0.20) \times 10^{14} \mathrm{~cm}^{-2}$ ] Figure 2(e) displays a zoomed-in view of the fitted FS contours of Figs. 2(a)-2(d) used to derive the carrier densities. For pristine $\mathrm{Yb}$-intercalated graphene (blue curve) the giant hole pocket assumes the shape of a flattened circle as the VHS extends along $\overline{\mathrm{KMK}}^{\prime}$. With increasing potassium dose its contour becomes more and more circular (purple and orange curves) until saturation sets in (yellow curve). The inset tracks the absolute changes $\left|\Delta k_{F}\right|$ of the Fermi wave vector along $\overline{\Gamma M}$ and $\overline{\Gamma K^{\prime}}$ relative to the pristine sample. While the initial evolution is restricted to the extended-VHS sector and the $\overline{\Gamma^{\prime}}$ direction remains basically unaffected, the hole pocket shrinks rather isotropically during the second deposition cycle. This suggests that surpassing the Fermi level pinning of the VHS is indeed the critical step in achieving the desired overdoping.

The energy-momentum cuts of Figs. 2(f)-2(h) reveal the evolution of the electronic structure in the vicinity of $\bar{M}$ towards $\bar{\Gamma}$ and $\bar{\Gamma}^{\prime}$ with increasing potassium dose [48]. The solid curves are parabolic fits to the spectral maxima and highlight the upward (downward) dispersion of the $\pi^{*}(\pi)$ band perpendicular to $\overline{\mathrm{KMK}}^{\prime}$ [49]. The changes in binding energy $E$ and absolute effective mass $\left|m^{*}\right|$ at $\overline{\mathrm{M}}$ of the 

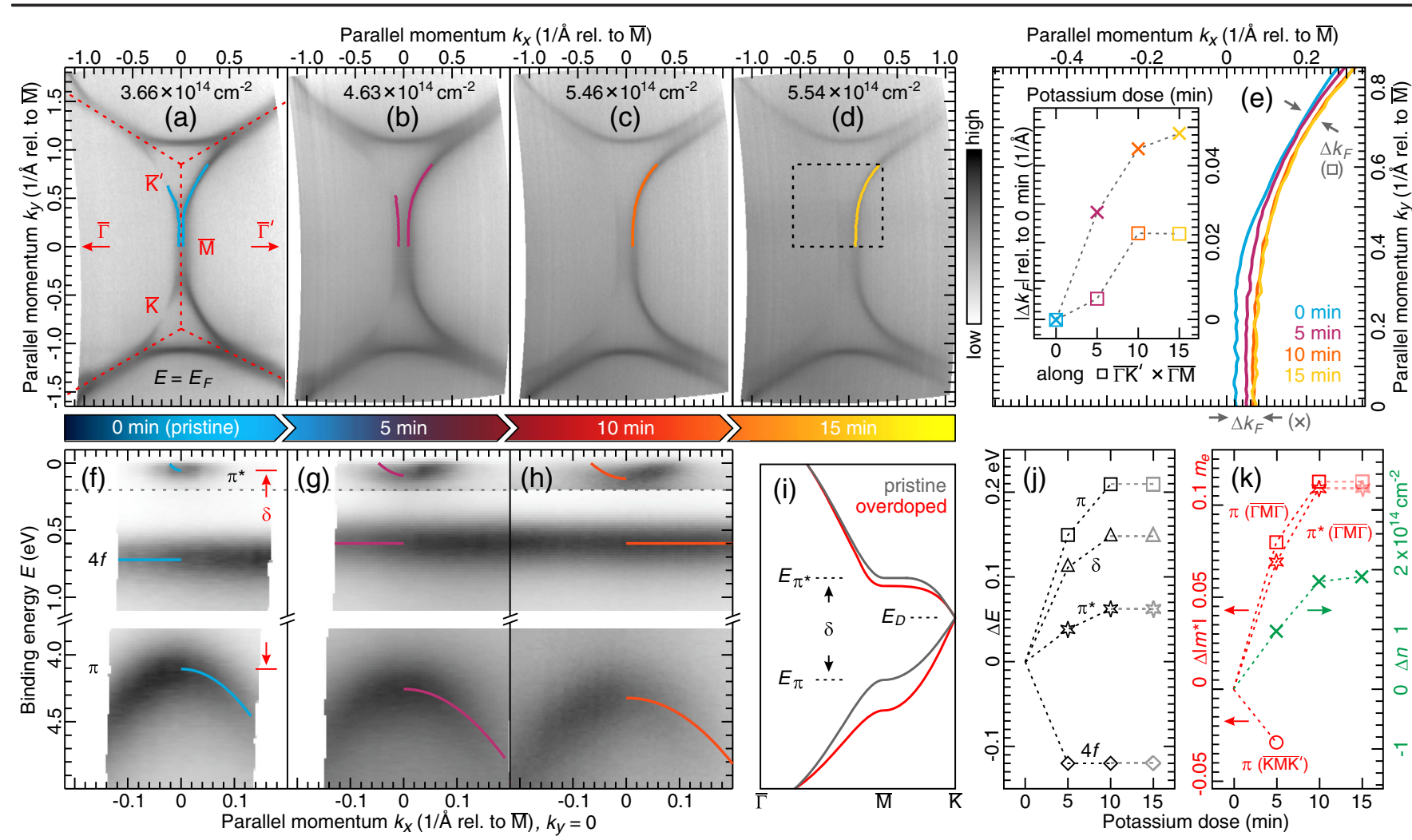

FIG. 2. (a)-(d) Fermi surfaces (logarithmic grayscale) and (f)-(h) energy-momentum cuts along $\overline{\Gamma M \Gamma}(h \nu=80 \mathrm{eV})$ for increasing potassium dose (color encoded). Solid curves are segments of fits to the data and grayscales differ between and within the individual panels to enhance the individual spectral features. (e) FS contours extracted from (a)-(d) within the dashed rectangle overlayed in (d). The inset shows the evolution $\left|\Delta k_{F}\right|$ of the Fermi wave vector along $\overline{\Gamma M}$ and $\overline{\Gamma K}$ relative to the pristine sample as a function of potassium dose. (i) Schematized $\pi$-band renormalizations upon overdoping (exaggerated for clarity). (j) Relative binding energy changes $\Delta E$ of $\pi, \pi^{*}, \mathrm{Yb} 4 f_{7 / 2}$ and the $\pi-\pi^{*}$ gap $\delta$ at $\overline{\mathrm{M}}$ with potassium dose. (k) Relative changes $\Delta$ in the charge carrier density $n$ (green symbols) and the absolute effective masses $\left|m^{*}\right|$ of $\pi, \pi^{*}$ at $\overline{\mathrm{M}}$ (red symbols). Transparent data points for 15 min exposure are extrapolated based on the saturation of $n$.

individual bands are plotted relative to the pristine sample in Figs. 2(j) and 2(k), respectively. With increasing potassium exposure the data quality degrades and the spectral weight of the $\pi$ bands is progressively quenched. In combination with the decreased energy resolution of $\approx 0.1 \mathrm{eV}$, errors of up to $50 \%$ (in particular for $m^{*}$ ) can result and we do not aim at an overly quantitative analysis. Yet our data support a consistent picture of $\pi$-band renormalizations in overdoped graphene [Fig. 2(i)], which will hopefully stimulate further theoretical modeling. First we note that the binding energy of the $\mathrm{Yb} 4 f_{7 / 2}$ state decreases upon potassium exposure, qualitatively matching the previously reported increase when reducing the $n$ doping away from the VHS [14]. In order to reach the extended van Hove scenario of Figs. 2(a) and 2(f), the $\pi^{*}$ band had to bend down considerably whereas the $\pi$ bandwidth $\left(E_{\pi}-E_{D} \approx 2.5 \mathrm{eV}\right)$ is only slightly lower than for intrinsic monolayer graphene on $\mathrm{SiC}(\approx 2.8 \mathrm{eV})$ [33]. As such the $\pi-\pi^{*}$ band gap at $\overline{\mathrm{M}}, \delta \approx 4.1 \mathrm{eV}$, has decreased compared to the tight-binding description relevant for lower doping levels. While both $E_{\pi}$ and $E_{\pi^{*}}$ increase with potassium doping [Fig. 2(j)], the $\pi$ band is much more affected, contrary to when QFMLG is initially doped up to the VHS. In consequence of this nonrigid band warping beyond a simple shift of $E_{F}, \delta$ has already expanded by more than $0.1 \mathrm{eV}$ following the first deposition cycle. That is, the trend of a reduced $\pi-\pi^{*}$ gap with increasing $n$ doping [13-15] reverses once QFMLG is overdoped past the VHS. The system thus counteracts the imposed overdoping and tries to keep the $\pi^{*}$ VHS close to $E_{F}$ by pushing the $\pi$ bands away from each other at $\bar{M}$ [Fig. 2(i)]. This scenario is substantiated by the apparent increase in $\left|m^{*}\right|$ (i.e., decrease in curvature) along $\overline{\Gamma \mathrm{M} \Gamma^{\prime}}$ for both bands [Fig. 2(k)]. In the orthogonal $\overline{\mathrm{KMK}}^{\prime}$ direction, the absolute effective mass $\left|m^{*}\right|$ of the $\pi$ band at $\bar{M}$ decreases, suggesting that the Dirac point energy $E_{D}$ does not move rigidly with $E_{\pi}$ [Fig. 2(i)]. At this point, a substantial downshift of $E_{D}$ together with the entire $\pi$-band system can also be excluded due to the basically constant size of the FS hole pocket in $\overline{\Gamma K}^{\prime}$ direction [Fig. 2(e)]. $E_{D}$ therefore appears to act as a relatively inert reference level of the renormalizing $\pi$-band structure near van Hove filling, consistent with QFMLG underdoped to below the VHS [14]. Although $n$ increases linearly over the first two deposition cycles [Fig. 2(k)], the 
$\pi$ bands and their effective masses along ${\overline{\Gamma M \Gamma^{\prime}}}^{\prime}$ seem to renormalize considerably less during the second exposure [50]. This could be explained by the fading influence of the VHS Fermi-level pinning, now that it has clearly been surpassed. In parallel, the FS evolves more isotropically [Fig. 2(e)] indicating a transition to a doping-induced shift of the entire $\pi$-band system instead of band warping which dominates across the Lifshitz transition. As the observed band structure renormalizations cannot be described in a single-particle picture [15,51] our experiments highlight the influence of nonlocal many-body effects in graphene under extreme $n$-type (over)doping.

In summary, epitaxial graphene on $\mathrm{SiC}$ is $n$ doped past the extended van Hove singularity, up to a carrier density of $5.5 \times 10^{14} \mathrm{~cm}^{-2}$, through a combination of ytterbium intercalation and potassium adsorption. Angle-resolved photoelectron spectroscopy confirms the overdoping by probing a shrinking hole pocket in the Fermi surface. The completion of the associated Lifshitz transition is driven by electronic structure renormalizations rather than a rigid band shift, exemplified by an increasing energy gap between the upper and lower $\pi$ bands at $\bar{M}$. Our results open up the previously inaccessible beyond-van-Hove regime in the phase diagram of graphene where a variety of exotic ground states may stabilize.

We would like to thank Helmholtz-Zentrum Berlin for the allocation of synchrotron radiation beamtime under proposals 182-07119-ST and 191-08299-ST/R. We are grateful to Kristen Kaasbjerg for providing valuable information on charged impurity scattering in doped graphene. This work was supported by the Deutsche Forschungsgemeinschaft (DFG) through Sta315/9-1.

"p.rosenzweig@ @ fkf.mpg.de

[1] C. Berger, Z. Song, T. Li, X. Li, A. Y. Ogbazghi, R. Feng, Z. Dai, A. N. Marchenkov, E. H. Conrad, P. N. First, and W. A. de Heer, J. Phys. Chem. B 108, 19912 (2004).

[2] K. S. Novoselov, A. K. Geim, S. V. Morozov, D. Jiang, M. I. Katsnelson, I. V. Grigorieva, S. V. Dubonos, and A. A. Firsov, Nature (London) 438, 197 (2005).

[3] A. K. Geim and K. S. Novoselov, Nat. Mater. 6, 183 (2007).

[4] M. I. Katsnelson, Mater. Today 10, 20 (2007).

[5] A. H. Castro Neto, F. Guinea, N. M. R. Peres, K. S. Novoselov, and A. K. Geim, Rev. Mod. Phys. 81, 109 (2009).

[6] T. O. Wehling, E. Şaşıŏglu, C. Friedrich, A. I. Lichtenstein, M. I. Katsnelson, and S. Blügel, Phys. Rev. Lett. 106, 236805 (2011).

[7] V. N. Kotov, B. Uchoa, V. M. Pereira, F. Guinea, and A. H. Castro Neto, Rev. Mod. Phys. 84, 1067 (2012).

[8] Y. Cao, V. Fatemi, K. Watanabe, T. Taniguchi, E. Kaxiras, and P. Jarillo-Herrero, Nature (London) 556, 43 (2018).

[9] Y. Cao, V. Fatemi, A. Demir, S. Fang, S. L. Tomarken, J. Y. Luo, J. D. Sanchez-Yamagishi, K. Watanabe, T. Taniguchi,
E. Kaxiras, R. C. Ashoori, and P. Jarillo-Herrero, Nature (London) 556, 80 (2018).

[10] A. Kerelsky, L. J. McGilly, D. M. Kennes, L. Xian, M. Yankowitz, S. Chen, K. Watanabe, T. Taniguchi, J. Hone, C. Dean, A. Rubio, and A. N. Pasupathy, Nature (London) 572, 95 (2019).

[11] X. Lu, P. Stepanov, W. Yang, M. Xie, M. A. Aamir, I. Das, C. Urgell, K. Watanabe, T. Taniguchi, G. Zhang, A. Bachtold, A. H. MacDonald, and D. K. Efetov, Nature (London) 574, 653 (2019).

[12] D. Marchenko, D. V. Evtushinsky, E. Golias, A. Varykhalov, Th. Seyller, and O. Rader, Sci. Adv. 4, eaau0059 (2018).

[13] J. L. McChesney, A. Bostwick, T. Ohta, Th. Seyller, K. Horn, J. González, and E. Rotenberg, Phys. Rev. Lett. 104, 136803 (2010).

[14] Ph. Rosenzweig, H. Karakachian, S. Link, K. Küster, and U. Starke, Phys. Rev. B 100, 035445 (2019).

[15] S. Link, S. Forti, A. Stöhr, K. Küster, M. Rösner, D. Hirschmeier, C. Chen, J. Avila, M. C. Asensio, A. A. Zakharov, T. O. Wehling, A. I. Lichtenstein, M. I. Katsnelson, and U. Starke, Phys. Rev. B 100, 121407(R) (2019).

[16] V. A. Khodel and V. R. Shaginyan, JETP Lett. 51, 553 (1990).

[17] K. Gofron, J. C. Campuzano, A. A. Abrikosov, M. Lindroos, A. Bansil, H. Ding, D. Koelling, and B. Dabrowski, Phys. Rev. Lett. 73, 3302 (1994).

[18] D. H. Lu, M. Schmidt, T. R. Cummins, S. Schuppler, F. Lichtenberg, and J. G. Bednorz, Phys. Rev. Lett. 76, 4845 (1996).

[19] V. Y. Irkhin, A. A. Katanin, and M. I. Katsnelson, Phys. Rev. Lett. 89, 076401 (2002).

[20] J. G. Storey, J. L. Tallon, and G. V. M. Williams, Phys. Rev. B 76, 174522 (2007).

[21] D. Yudin, D. Hirschmeier, H. Hafermann, O. Eriksson, A. I. Lichtenstein, and M. I. Katsnelson, Phys. Rev. Lett. 112, 070403 (2014).

[22] I. Lifshitz, Sov. Phys. JETP 11, 1130 (1960).

[23] G. E. Volovik, Low Temp. Phys. 43, 47 (2017).

[24] I. F. Herbut, Phys. Rev. Lett. 97, 146401 (2006).

[25] C. Honerkamp, Phys. Rev. Lett. 100, 146404 (2008).

[26] R. Nandkishore, L. S. Levitov, and A. V. Chubukov, Nat. Phys. 8, 158 (2012).

[27] M. L. Kiesel, C. Platt, W. Hanke, D. A. Abanin, and R. Thomale, Phys. Rev. B 86, 020507(R) (2012).

[28] A. M. Black-Schaffer and C. Honerkamp, J. Phys. Condens. Matter 26, 423201 (2014).

[29] D. Makogon, R. van Gelderen, R. Roldán, and C. M. Smith, Phys. Rev. B 84, 125404 (2011).

[30] S. Raghu, X.-L. Qi, C. Honerkamp, and S.-C. Zhang, Phys. Rev. Lett. 100, 156401 (2008).

[31] K. V. Emtsev, A. Bostwick, K. Horn, J. Jobst, G. L. Kellogg, L. Ley, J. L. McChesney, T. Ohta, S. A. Reshanov, J. Röhrl, E. Rotenberg, A. K. Schmid, D. Waldmann, H. B. Weber, and Th. Seyller, Nat. Mater. 8, 203 (2009).

[32] Helmholtz-Zentrum Berlin für Materialien und Energie, J. Large-Scale Res. Facil. 4, A128 (2018).

[33] A. Bostwick, T. Ohta, Th. Seyller, K. Horn, and E. Rotenberg, Nat. Phys. 3, 36 (2007).

[34] C. Hwang, D. Y. Kim, D. A. Siegel, K. T. Chan, J. Noffsinger, A. V. Fedorov, M. L. Cohen, B. Johansson, J. B. Neaton, and A. Lanzara, Phys. Rev. B 90, 115417 (2014). 
[35] W.-K. Tse and S. Das Sarma, Phys. Rev. Lett. 99, 236802 (2007).

[36] S. Forti, K. V. Emtsev, C. Coletti, A. A. Zakharov, C. Riedl, and U. Starke, Phys. Rev. B 84, 125449 (2011).

[37] Using a parabolic bare band and the algorithm of: I. Pletikosić, M. Kralj, M. Milun, and P. Pervan, Phys. Rev. B 85, 155447 (2012).

[38] B. M. Ludbrook, G. Levy, P. Nigge, M. Zonno, M. Schneider, D. J. Dvorak, C. N. Veenstra, S. Zhdanovich, D. Wong, P. Dosanjh, C. Straßer, A. Stöhr, S. Forti, C. R. Ast, U. Starke, and A. Damascelli, Proc. Natl. Acad. Sci. U.S.A. 112, 11795 (2015).

[39] T. Valla, A. V. Fedorov, J. Lee, J. C. Davis, and G. D. Gu, Science 314, 1914 (2006).

[40] I. M. Vishik, W. S. Lee, R. H. He, M. Hashimoto, Z. Hussain, T. P. Devereaux, and Z.-X. Shen, New J. Phys. 12, 105008 (2010).

[41] A. V. Fedorov, N. I. Verbitskiy, D. Haberer, C. Struzzi, L. Petaccia, D. Usachov, O. Y. Vilkov, D. V. Vyalikh, J. Fink, M. Knupfer, B. Büchner, and A. Grüneis, Nat. Commun. 5, 3257 (2014).
[42] K. Kaasbjerg and A.-P. Jauho, Phys. Rev. B 100, 241405(R) (2019); K. Kaasbjerg (private communication).

[43] J. M. Luttinger and J. C. Ward, Phys. Rev. 118, 1417 (1960).

[44] J. M. Luttinger, Phys. Rev. 119, 1153 (1960).

[45] The vertical $\overline{K M K}^{\prime}$ BZ border does not perfectly coincide with $k_{x}=0$ in experiment. The fitted contours [Figs. 2(a)2(e)] are corrected for these deviations considering the FS symmetry relative to $\overline{\mathrm{KMK}}^{\prime}$.

[46] T. Ohta, A. Bostwick, Th. Seyller, K. Horn, and E. Rotenberg, Science 313, 951 (2006).

[47] I. Gierz, J. Henk, H. Höchst, C. R. Ast, and K. Kern, Phys. Rev. B 83, 121408(R) (2011).

[48] Corresponding data for $15 \mathrm{~min}$ exposure not available.

[49] In Figs. 2(f)-2(h), the fits to the $\pi^{*}$ band adopt fixed $E_{F}$ crossings according to the FS fits of Figs. 2(a)-2(c).

[50] Data along $\overline{\mathrm{KMK}}^{\prime}$ not available.

[51] S. Ulstrup, M. Schüler, M. Bianchi, F. Fromm, C. Raidel, Th. Seyller, T. Wehling, and P. Hofmann, Phys. Rev. B 94, 081403(R) (2016). 